\section{$\varepsilon_{8: 0}$}

- Additional material is published online only. To view please visit the journal online (http://dx.doi.org/10.1136/ thoraxjnl-2016-209575).

Division of Pulmonary, Critical Care, and Sleep Medicine, Westchester Medical Center at New York Medical College, Valhalla, New York, USA

\section{Correspondence to}

Dr Aparna Kadambi, Division of Pulmonary, Critical

Care, and Sleep Medicine, Westchester Medical Center at New York Medical College, 100 Woods Road, Valhalla, New York 10595, USA; draparnakadambi@gmail.com

Received 20 October 2016 Revised 28 May 2017 Accepted 5 June 2017 Published Online First 29 July 2017

\title{
Double whammy: the disappearing right atrium
}

\author{
Aparna Kadambi, Parimal Kumar Chaudhari, Lawrence DeLorenzo, Oleg Epelbaum
}

A 66-year-old man with hepatitis $\mathrm{C}$ virus (HCV) infection complicated by liver cirrhosis presented with about 6 months of severe right shoulder pain as well as more recent progressive dyspnoea on exertion and lower extremity oedema. On outpatient evaluation at the onset of shoulder symptoms, they had been ascribed to an orthopaedic aetiology. $\mathrm{He}$ had recently completed treatment for $\mathrm{HCV}$ with ledipasvir/sofosbuvir after failure to respond to a regimen of interferon/ribavirin. Approximately 2 years earlier, he had been diagnosed with unresectable multifocal stage III hepatocellular carcinoma (HCC) and treated with transarterial chemoembolisation (TACE), radiofrequency ablation and ethanol ablation as a bridge to possible liver transplantation. On presentation, he was hemodynamically stable with no reported pulsus paradoxus. Physical examination revealed an elevated jugular venous pulse, anasarca and decreased breath sounds over the right lower chest. There was no clinically overt ascites. His plain chest radiograph showed a right pleural effusion and a thickened right paratracheal stripe (figure 1A). Subsequent contrast-enhanced CT of the chest confirmed a right pleural effusion and paratracheal lymphadenopathy. It also revealed elevation of the right hemidiaphragm as well as a large mass extending from the dome of the liver into the chest with near-total obliteration of the right atrial (RA) cavity by the combination of mass effect and an intracavitary filling defect (figure 1B). A similar filling defect was also present in the inferior vena cava. MRI of the abdomen with gadolinium enhancement performed about 3 months prior to the current presentation recapitulated analogous findings and showed invagination of the mass into the RA (figure 2). Echocardiographic images obtained via the transthoracic approach demonstrated a

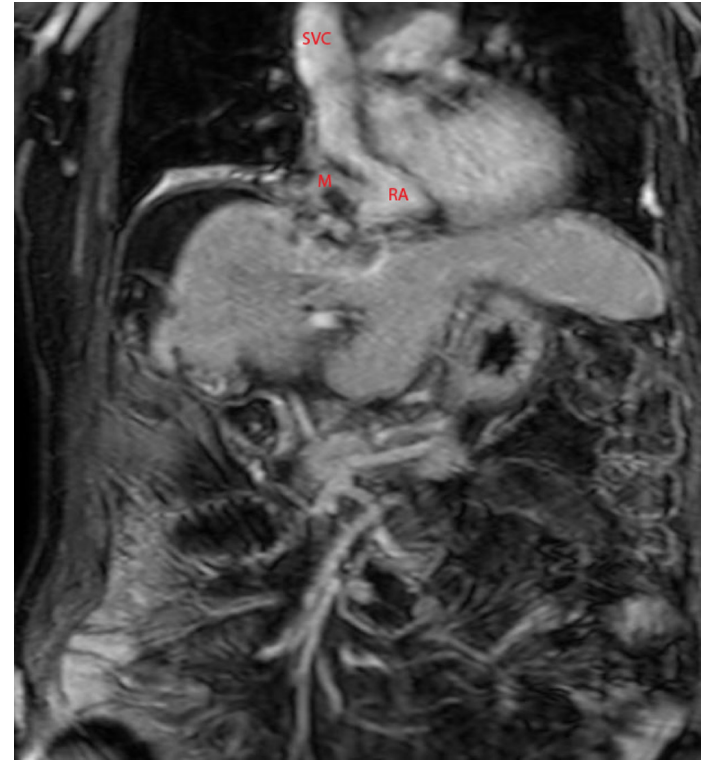

Figure 2 Coronal reconstruction of gadoliniumenhanced MRI of the abdomen demonstrating the transdiaphragmatic extension of the mass $(\mathrm{M})$ and the adjacent filling defect in the right atrium (RA). SVC denotes superior vena cava.

large intraluminal echodensity within the right atrium consistent with an intra-atrial mass, best illustrated on the four-chamber view (figure 3, see online Supplementary video). It was concluded that the patient suffered from transdiaphragmatic invasion by recurrent HCC resulting in referred right shoulder pain and extrinsic RA compression. That, combined with tumour thrombus extending into the RA by way of the hepatic venous system and the inferior vena cava (IVC), caused impaired
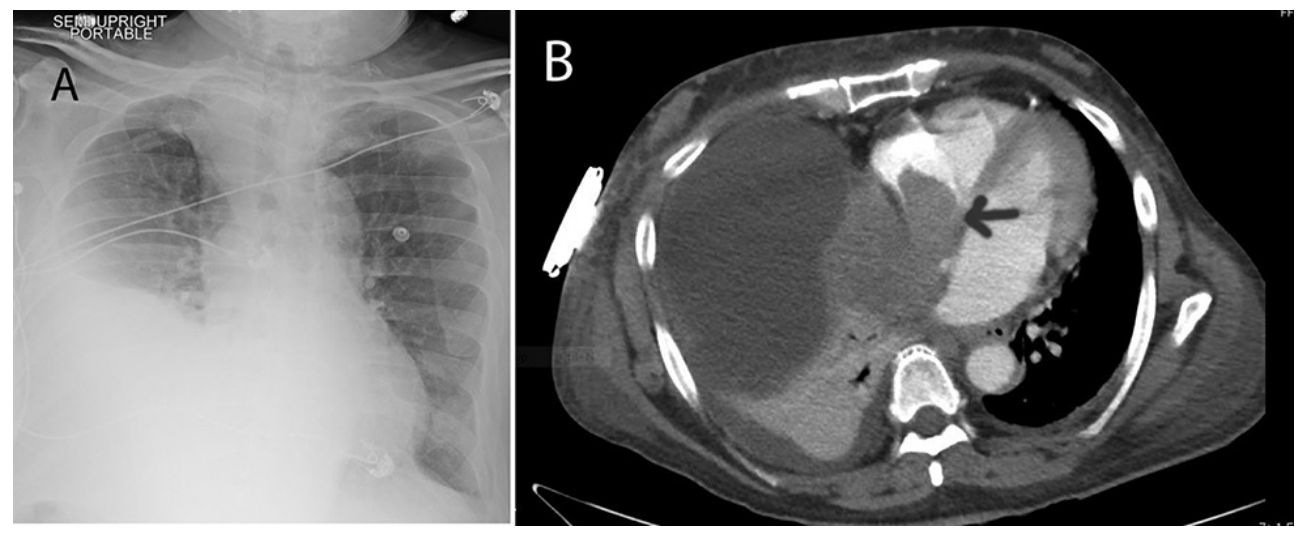

Figure 1 (A) Plain chest radiograph showing a right pleural effusion and paratracheal stripe widening. (B) Axial chest $\mathrm{CT}$ image showing a soft tissue mass abutting right atrium, the cavity of which contains an intraluminal hypodensity (arrow). A right pleural effusion and ascitic fluid are also visible. 


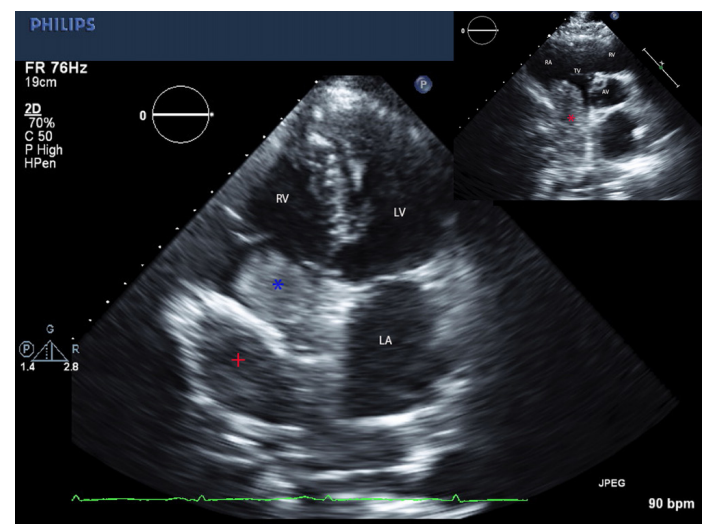

Figure 3 Four-chamber echocardiographic view showing a large intraluminal echodensity within the RA consistent with an intra-atrial mass (asterisk) as well as extrinsic compression of the RA by the mass (plus). Inset: Echocardiographic view showing tumour thrombus (asterisk) extending into RA by way of the inferior vena cava. AV, aortic valve; $L A$, left atrium; $L V$, left ventricle; $R A$, right atrium; $R V$, right ventricle; TV, tricuspid valve.

venous return and anasarca. His dyspnoea improved only modestly after drainage of the transudative right pleural effusion consistent with hepatic hydrothorax. The patient was deemed unfit for further therapeutic interventions and was discharged home with hospice services.

$\mathrm{HCV}$ is second only to hepatitis B as the most frequent cause of HCC worldwide. ${ }^{1}$ Extrahepatic spread of HCC occurs in a minority of patients $(15 \%-35 \%)$ and almost exclusively in those with locally advanced disease. ${ }^{23}$ Lung is the most common site of distant haematogenous dissemination of HCC. ${ }^{23}$ This malignancy has a propensity for growth into the hepatic venous system, but intravascular tumour extension into the RA via the IVC is a rare occurrence, though certainly not as exceptional as primary cardiac tumours, which are also part of the differential diagnosis of an intra-atrial mass. ${ }^{4}$ It is worth noting that cardiac tumour thrombus can be the initial presenting manifestation of HCC. ${ }^{5}$
Vascular and cardiac occlusion caused by such intraluminal filling by HCC can lead to secondary Budd-Chiari syndrome and haemodynamic compromise due to diminished venous return. Retrospective data suggest that carefully selected patients with resectable primary tumours can achieve 1-year survival rates close to $70 \%$ following partial hepatectomy plus thrombectomy for HCC with this complication. ${ }^{6}$ Those treated with TACE or symptomatic therapies have historically fared markedly worse.

Our case illustrates how HCC, a common abdominal neoplasm, can have a major impact on cardiopulmonary physiology through extension into the thorax by two routes: transdiaphragmatic and endovascular, thus placing the RA in peril both intrinsically and extrinsically. It also serves as a reminder that unremitting shoulder discomfort may herald grave diaphragmatic pathology.

\section{Competing interests None declared}

\section{Patient consent Obtained.}

Provenance and peer review Not commissioned; externally peer reviewed.

(c) Article author(s) (or their employer(s) unless otherwise stated in the text of the article) 2018. All rights reserved. No commercial use is permitted unless otherwise expressly granted.

\section{REFERENCES}

1 Perz JF, Armstrong GL, Farrington LA, et al. The contributions of hepatitis B virus and hepatitis $C$ virus infections to cirrhosis and primary liver cancer worldwide. J Hepatol 2006;45:529-38.

2 Uka K, Aikata H, Takaki S, et al. Clinical features and prognosis of patients with extrahepatic metastases from hepatocellular carcinoma. World J Gastroenterol 2007:13:414-20.

3 Katyal S, Oliver JH, Peterson MS, et al. Extrahepatic metastases of hepatocellular carcinoma. Radiology 2000;216:698-703.

4 Kato Y, Tanaka N, Kobayashi K, et al. Growth of hepatocellular carcinoma into the right atrium. Report of five cases. Ann Intern Med 1983;99:472-4.

5 Sun JH, Zhang YL, Nie CH, et al. Long-term survival after chemoembolization of metastatic right atrial tumor thrombus as a presenting feature of hepatocellular carcinoma: A case study. Oncol Lett 2012;3:975-7.

6 Wang Y, Yuan L, Ge RL, et al. Survival benefit of surgical treatment for hepatocellular carcinoma with inferior vena cava/right atrium tumor thrombus: results of a retrospective cohort study. Ann Surg Oncol 2013;20:914-22. 